\title{
ESTADO NUTRICIONAL DE CRIANÇAS E ADOLESCENTES DE UMA ESCOLA DE FUTEBOL DA ZONA SUL DE SÃO PAULO
}

\author{
Dayane Passos de Oliveira BAIA \\ Luana de Almeida RODRIGUES
}

Maria Lúcia Ramos Pereira COSTA

Nyvian Alexandre KUTZ

Victor Alexandre Ferreira TARINI

Marcia Maria Hernandes de Abreu de Oliveira SALGUEIRO

${ }^{1}$ Nutricionista, Centro Universitário Adventista de São Paulo, dayane.passos.o@ gmail.com
${ }^{2}$ Nutricionista, Centro Universitário Adventista de São Paulo, luana_rodris@ hotmail.com
${ }^{3}$ Nutricionista, Centro Universitário Adventista de São Paulo, lucia_rcosta@ @otmail.com
${ }^{4}$ Nutricionista, Centro Universitário Adventista de São Paulo, ny_adv@ @otmail.com
${ }^{5}$ Doutor, Centro Universitário Adventista de São Paulo, victor.tatini@gmail.com
${ }^{6}$ Doutora, Centro Universitário Adventista de São Paulo, marciasalgueironutricionista@yahoo.com.br

Recebido em: 24/05/2016 - Aprovado em: 30/11/2017 - Disponibilizado em: 30/12/2017

\begin{abstract}
RESUMO
Avaliou-se o estado nutricional de crianças e adolescentes de uma escola de futebol. Estudo transversal com 80 crianças e adolescentes do sexo masculino, de 5 a 15 anos. Foram coletados nome, idade e informações necessárias à classificação econômica. A avaliação do estado nutricional ocorreu com a aferição das medidas de peso, estatura, circunferência do braço e dobras cutâneas tricipital e subescapular. As associações entre idade e classe econômica e dados antropométricos foram analisadas pelo teste de Kruskal-Wallis, seguido do teste "post hoc" de Dunn, com intervalo de confiança (IC) de 95\%. Em todos os casos, foram consideradas como estatisticamente diferentes as médias quando $\mathrm{p}<0,05.94 \%$ dos meninos com IMC adequado e apenas 5\% com excesso de peso. A classe econômica predominante é a B. Observa-se aumento significante de massa magra entre as faixas etárias 11-12 e 13-14 anos. Encontra-se predomínio de eutrofia e classe econômica alta. Os meninos de classe econômica mais baixa encontram-se com peso mais elevado quando comparados aos de classe econômica mais alta.
\end{abstract}

Palavras-chave: Criança. Adolescente. Futebol.Antropometria. Estado Nutricional.

\begin{abstract}
We evaluated the nutritional status of children and adolescents in a football school. Cross-sectional study with 80 children and male adolescents, 5 to 15 years. They collected name, age and information necessary for economic classification. The assessment of nutritional status occurred in the measurement of weight, height, arm circumference and triceps and subscapular skinfold. The associations between age and economic class, and anthropometric data were analyzed using the Kruskal-Wallis test followed by "post hoc" Dunn, with a confidence interval (CI) of 95\%. In all cases, they were considered as statistically different averages when $\mathrm{p}<0,05$. $94 \%$ of boys with adequate BMI and only $5 \%$ overweight. The dominant economic class is B. observed a significant increase in lean body mass between age groups 11-12 and 13-14 years. It is the predominance of eutrophic and high economic class. The lower economic class boys are with higher weight as compared to higher economic class.
\end{abstract}

Keywords:Children. Adolescent. Soccer.Anthropometry. Nutritional Status. 


\section{INTRODUÇÃO}

A antropometria é um método que permite a avaliação do peso, da altura e de outras medidas do corpo humano, representando um importante recurso para a avaliação do estado nutricional do indivíduo e ainda oferece dados para o acompanhamento do crescimento e desenvolvimento de crianças e adolescentes (BRASIL, 2004). O método mais utilizado para determinar o estado nutricional é o índice de massa corporal (IMC) que é a relação do peso em quilogramas pela estatura em metros elevada ao quadrado. Os pontos de cortes estabelecidos para crianças, adolescentes e adultos foram retirados do relatório do World Health Organization (WHO, 1995) e recomendados pelo Ministério da Saúde (BRASIL, 2004).

\section{A International Obesity Task Force} (IOTF), em 1997, propôs definir o estado nutricional dos menores de 20 anos com base nos diagnósticos de desnutrição, excesso de peso e obesidade e/ou na alteração de diversos indicadores bioquímicos associados a doenças crônicas na fase adulta. Em 2000, foi publicado o conjunto de valores críticos para definir excesso de peso e obesidade no grupo etário de 2 a 18 anos. O uso das curvas baseadas no IMC para idade para definição do estado nutricional em crianças e adolescentes propiciou, por um lado, soluções práticas e, por outro lado, debates sobre o uso dessas curvas para avaliação do estado nutricional de grupos em crescimento (CONDE; MONTEIRO, 2006).

Vários estudos com crianças e adolescentes têm demonstrado o benefício da atividade física no estímulo ao crescimento e desenvolvimento, prevenção da obesidade, incremento da massa óssea, aumento da sensibilidade à insulina, melhora do perfil lipídico, diminuição da pressão arterial (BRODERICK; WINTER; ALLAN, 2006), desenvolvimento da socialização e da capacidade de trabalhar em equipe (AZEVEDO et al., 2007).

Assim, o objetivo desta pesquisa foi avaliar o estado nutricional de crianças e adolescentes que praticam atividade esportiva numa escola de futebol na zona sul de São Paulo, caracterizar a população segundo as variáveis demográficas, idade, classe econômica e relacionar o estado nutricional com a classe econômica.

\section{MÉTODO}

Trata-se de um estudo transversal com crianças e adolescentes do sexo masculino, de 05 a 15 anos, que praticam futebol em uma escola no bairro Vila Sofia naZona Sul de São Paulo. Os dados foram coletados entre os meses de setembro e outubro de 2012 na própria escola. O trabalho foi aprovado pelo Comitê de Ética em pesquisa do UNASP com protocolo 46744 em 14 de junho de 2012.

Para realização da pesquisa foram convidados 300 meninos, para os quais foi 
distribuído o Termo de Consentimento Livre e Esclarecido em duas vias, no qual esclarecia os objetivos do trabalho. Orientou-se que a participação na pesquisa era voluntária. Compareceram à coleta de dados 100 meninos. Somente 80 entregaram o Termo de Consentimento Livre e Esclarecido assinado pelos responsáveis.

Os dados pessoais como nome, idade e informações necessárias à classificação econômica foram coletados por meio de entrevista individual. Crianças abaixo de 10 anos estavam acompanhadas dos pais ou responsáveis durante a entrevista. O Critério de Classificação Econômica Brasil, enfatiza o poder de compra das pessoas e famílias urbanas, abandonando a pretensão de classificar a população em termos de "classes sociais", e sim classe econômica. Relaciona a escolaridade do chefe da família e os itens de posse como geladeira, televisão, automóvel, máquina de lavar, entre outros; estabelecendo pontos de acordo com a quantidade de cada item. A partir desses valores classifica-se a classe econômica em oito classes: A1, A2, B1, B2, C1, C2, D e E; sendo as classes A com maior poder de compra e a $E$ com menor (ABEP, 2010).

A avaliação do estado nutricional foi realizada com a aferição das medidas de peso, estatura, circunferência do braço e dobras cutâneas tricipital e subescapular. A medida de peso foi realizada utilizando-se balança portátil digital eletrônica marca Plenna ${ }^{\circledR}$, onde os meninos foram solicitados a ficarem com o mínimo de roupa possível e descalços. Para a aferição da estatura, utilizou-se estadiômetro portátil com altura máxima de 2,30 metros, com indivíduos descalços, os pés paralelos, os tornozelos juntos, em posição ereta e os braços estendidos ao longo do corpo, com a cabeça posicionada conforme o Plano de Frankfurt (JELLIFFE, 1968). Utilizaram-se as medidas de peso e estatura para o cálculo do IMC, que é a relação do peso em quilogramas pela estatura em metros elevada ao quadrado (ONIS et al., 2007).

Para aferição da circunferência do braço direito foi utilizada fita métrica inelástica posicionada no ponto médio entre o acrômio e olécrano, com o braço a ser medido flexionado em direção ao tórax. Após isso, solicitou-se que os meninos estendessem o braço ao longo do corpo, com a palma da mão voltada para a coxa. No ponto marcado, contornou-se o braço com a fita métrica flexível de forma ajustada, evitando compressão da pele ou folga (BRITO; DREYER, 2012).

As dobras cutâneas medidas foram a tricipital e a subescapular (ONIS et al, 2007).Para medição da adiposidade corporal foi utilizado um plicômetroSlimGuide, graduado em 1,0 mm (HEYWARD; GIBSON, 1991). Cada dobra cutânea foi medida três vezes e o valor médio foi utilizado para o cálculo. Para a aferição da dobra cutânea tricipital foi utilizada a seguinte 
técnica: os meninos foram orientados a deixarem o braço direito solto e relaxado; e com uma fita métrica, mediu-se o comprimento entre o processo acromial da escápula e o olécrano, e foi marcado o ponto médio; aproximadamente $2 \mathrm{~cm}$ acima deste ponto, pinçou-se a pele sobre o tríceps, entre o polegar e o indicador; puxou-se a pele ligeiramente, afastando-a do músculo; após pinçar a pele entre as extremidades do plicômetroSlimGuide, no ponto marcado, leuse a medida que o plicômetro acusava em milímetros (mm). Para a aferição da dobra subescapular foi medido e marcado um ponto $1 \mathrm{~cm}$ abaixo do ângulo inferior da escápula, com o braço direito em extensão, utilizando o plicômetroSlimGuide. Todas as medidas foram realizadas três vezes, e a média dos valores foi utilizada para os cálculos (VANNUCCHI; UNAMUNO; MARCHINI, 1996). A coleta dos dados foi feita por uma equipe de técnicos previamente treinada para aferição das medidas antropométricas (peso, altura e circunferência do braço, dobras cutâneas triciptal e subescapular).

A classificação do estado nutricional de acordo com o IMC seguiu as recomendações de Cole et al, em baixo peso os meninos com IMC abaixo do percentil 5; peso adequado meninos com IMC entre os percentis 5 e 85; excesso de peso entre os percentis 85 e 95; e os meninos com IMC acima do percentil 95 foram classificados como obesidade (EISENSTEINet al., 2000).
A circunferência do braço e as dobras cutâneas foram agrupadas para obter o resultado da adiposidade corporal. A Soma das dobras cutâneas triciptal e subescapular superior ao percentil 90 foi classificada em excesso de adiposidade. Foi considerado excesso de adiposidade, percentil $>90$ e baixa adiposidade percentil < 10 (VITOLO et al., 2007).

A adiposidade corporal foi determinada por meio das seguintes variáveis: percentual de gordura corporal $(\% \mathrm{G})$ e massa corporal livre de gordura (MM). O percentual de gordura foi estimado usando as equações de Slaughteret al.(1988) por meio do somatório ( $\Sigma 2 \mathrm{DC})$ das dobras tricipital (TR) e subescapular (SE) como segue: para os meninos com $\Sigma 2 \mathrm{DC}$ menor que $35 \mathrm{~mm}$, utilizou-se a seguinte fórmula:

$\% G=1,21 x(T R+S E)-0,008 x(T R+S E) 2-1,7$

Para os meninos com $\Sigma 2$ DC igual ou superior a $35 \mathrm{~mm}$ utilizou-se a equação:

$$
\% G=0,783 x(T R+S E)+1,6(15)
$$

Após a obtenção do percentual de gordura, os meninos foram divididos em quatro grupos segundo o percentual de gordura: magros: percentual de gordura < 10\%; adiposidade adequada: percentual de gordura $\geq 10 \%$ até $\leq 20 \%$; excesso de gordura: percentual de gordura $>20 \%$ e < 25\%; obesidade: percentual de gordura $\geq$ $25 \%$. Após o cálculo do percentual de gordura, o peso da gordura foi calculado da seguinte maneira: 


\section{$\frac{\% G \times \text { peso corporal }}{100}$}

A massa livre de gordura foi calculada subtraindo-se a massa de gordura do peso corporal (LOHMAN, 1987).

Para análise dos dados, a amostra foi subdividida em faixas etárias: a primeira compreendeu as idades de 5 a 9 anos, e a segunda, de 10 a 15 anos. Para análise descritiva foi utilizado o programa de computador Microsoft Office Excel, versão 2007. Os resultados foram expressos em médias e desvios-padrão. A seguir, utilizou-se o método de D'Agostino e Pearson para se testar a normalidade dos dados. O teste de D'Agostino e Pearson mostrou que as variáveis em estudo se mostraram não paramétricas. Foram, então, determinadas as associações entre idade e classe econômica e dados antropométricos. As classes econômicas foram agrupadas em A (A1 e A2), B (B1 e B2) e C (C1 e C2). Foram feitas por meio da análise de variância "oneway" para dados não paramétricos, segundo o teste de Kruskal-Wallis, seguido do teste "post hoc" de Dunn, com intervalo de confiança (IC) de 95\%. Em todos os casos, foram consideradas como estatisticamente diferentes as médias quando $\mathrm{p}<0,05$. As análises estatísticas foram feitas por meio do pacote estatístico GraphPadPrism, versão 6.0 para Windows.

\section{RESULTADOS}

Foram avaliados 80 meninos de 5 a 15 anos de idade, divididos entre crianças de 5 a $9 \operatorname{anos}(26,25 \%)$ e adolescentes de 10 a 15 anos $(73,75 \%)$, dados encontrados na Tabela 1. A classe econômica predominante foi a B (B1 e B2) com $48,75 \%$.

Tabela 1: Características sociodemográficas de meninos de uma escola de futebol, zona sul de São Paulo.

\begin{tabular}{ccc}
\hline Faixa etária & n & $\%$ \\
\hline 5 a 9 anos & 21 & 26,25 \\
$\mathbf{1 0}$ a 15 anos & 59 & 73,75 \\
\hline Classe econômica & & \\
\hline A (A1 e A2) & 17 & 21,25 \\
B (B1 e B2) & 39 & 48,75 \\
C (C1 e C2) & 24 & 30
\end{tabular}

Fonte: Dados da pesquisa dos autores

Na Tabela 2 observa-se a relação em número e porcentagem de meninos de acordo com estatura, peso, IMC e o percentual de gordura corporal. A maior parte dos meninos encontra-se com peso e estatura adequados para sua idade (95\% e 97\%, respectivamente). Em relação ao IMC apenas $1 \%$ apresenta-se com IMC baixo, $5 \%$ com obesidade e nenhum menino com excesso de peso. Quanto ao percentual de gordura corporal verifica-se que $10 \%$ foram classificados em magreza, $15 \%$ em obesidade e $8 \%$ em excesso de gordura. Observa-se que 67\% apresentam-se com a percentual de gordura corporal adequada, 
contrastando com a porcentagem de adequação para o IMC que foi de $94 \%$.

Tabela 2: Distribuição dos meninos de acordo com as diferentes categorias antropométricas. Escola de futebol, zona sul de São Paulo.

\begin{tabular}{|c|c|c|}
\hline \multirow{2}{*}{$\begin{array}{l}\text { CATEGORIA } \\
\text { Antropometria }\end{array}$} & \multicolumn{2}{|c|}{ PARÂMETRO } \\
\hline & Estatura & Peso \\
\hline Percentil 3 & $4(5 \%)$ & $2(2 \%)$ \\
\hline Percentil 3-97 & $76(95 \%)$ & $77(97 \%)$ \\
\hline Percentil 97 & $1(1,25 \%)$ & $1(1 \%)$ \\
\hline \multicolumn{3}{|l|}{ IMC } \\
\hline Percentil $\leq 5$ & $1(1,25 \%)$ & Baixo \\
\hline Entre Percentil 50 e 85 & $\begin{array}{l}75 \\
(93,75 \%)\end{array}$ & Adequado \\
\hline Percentil 95 & $0(0 \%)$ & $\begin{array}{l}\text { Excesso de } \\
\text { peso }\end{array}$ \\
\hline Percentil $\geq 95$ & $4(5 \%)$ & Obesidade \\
\hline \multicolumn{3}{|l|}{ \% Gordura Corporal* } \\
\hline$<10 \%$ & $8(10 \%)$ & Magro \\
\hline$<20 \%$ & $52(67 \%)$ & Adequado \\
\hline$<25 \%$ & $6(8 \%)$ & $\begin{array}{l}\text { Excesso de } \\
\text { gordura }\end{array}$ \\
\hline$\geq 25 \%$ & $12(15 \%)$ & Obesidade \\
\hline
\end{tabular}

$\mathrm{Na}$ Figura 1 são apresentadas as medidas antropométricas em relação a idade. Observa-se que o aumento da estatura e do peso foi significante nas faixas etárias de 1112 anos para 13-14-anos (média \pm DP) $\mathrm{p}<$ 0,05. Nas variáveis IMC $\left(\mathrm{kg} / \mathrm{m}^{2}\right)$, dobras cutâneas e percentual de gordura não foram encontradas nenhuma diferença estatisticamente significante. No entanto, quanto a massa magra $(\mathrm{kg})$ houve aumento significante entre as faixas etárias dos 11-12 e 13-14 anos (média \pm DP) $p<0,05$.

Figura 1: Características dos meninos de acordo com Estatura $(\mathrm{cm})$, Peso Corporal $(\mathrm{Kg})$, Índice de Massa Corporal $\left(\mathrm{kg} / \mathrm{m}^{2}\right)$, Dobras Cutâneas $(\mathrm{mm}), \%$ de Gordura e Massa Magra $(\mathrm{kg})$ em relação à idade. Escola de futebol, zona sul de São Paulo. O símbolo estatístico $(*)$ revela a diferença significante $\mathrm{p}<0,05$.

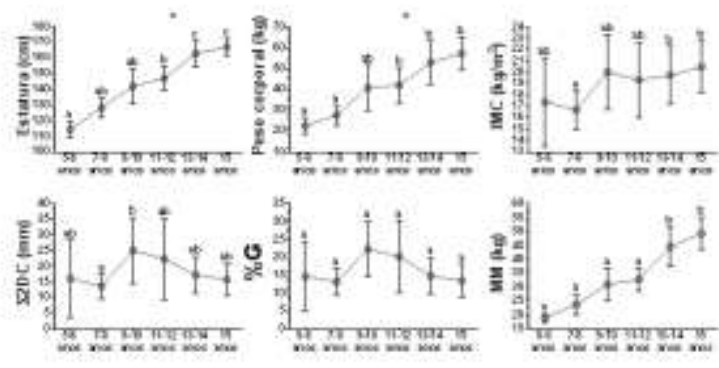

Fonte: Dados da pesquisa dos autores

A Figura 2 apresenta as medidas antropométricas dos meninos em relação à classe econômica. Foram observadas diferenças estatisticamente significantes apenas para o peso $(\mathrm{kg})$, onde se verifica aumento entre os meninos da classe $\mathrm{C}$, em comparação aos das classes A e B. 
Figura 2: Características dos meninos de acordo com Estatura (cm), Peso Corporal (Kg), Índice de Massa Corporal $\left(\mathrm{kg} / \mathrm{m}^{2}\right)$, Dobras Cutâneas $(\mathrm{mm})$, \% de Gordura e Massa Magra $(\mathrm{kg})$ em relação à classe econômica. Escola de futebol, zona sul de São Paulo. O símbolo estatístico (*) revela a diferença significante $\mathrm{p}<0,05$.
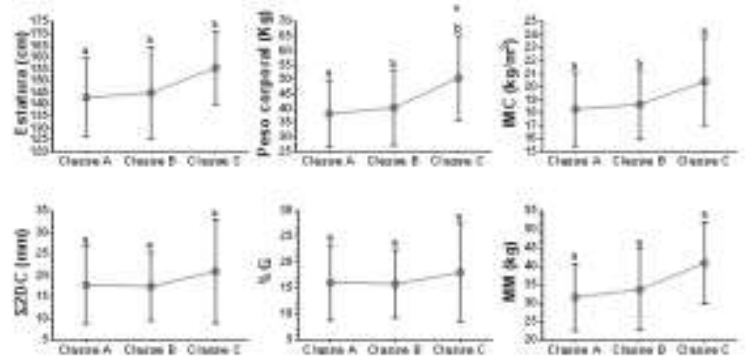

Fonte: Dados da pesquisa dos autores

\section{DISCUSSÃO}

O presente estudo avaliou o estado nutricional de crianças e adolescentes de uma escola de futebol da zona sul de São Paulo. A maior parte dos meninos pesquisados encontra-se com IMC adequado, sendo assim classificados como eutróficos. Isso se deve provavelmente a prática de atividade física regular, a qual promove gasto energético intenso, facilitando o controle ponderal, melhorando assim o estado nutricional. $\mathrm{O}$ estudo de Ulbrich et al. (2007) também avaliou jovens praticantes de atividades desportivas, de ambos os sexos, sendo classificados como eutróficos.

A participação da criança e do adolescente em esportes sistematizados é importante na busca de benefício físico e social (ULBRICH et al.,2007). Uma possível explicação para os achados do presente estudo está no fato de a atividade física regular impactar positivamente a composição corporal, por meio da redução da gordura e consequentemente da massa corporal total (SALES; MOREIRA, 2012).

Com relação aos aspectos antropométricos, observou-se, como esperado aumento da estatura, peso corporal, IMC, circunferência do braço e dobras cutâneas, em função do aumento da idade, refletindo as influências hormonais para essa faixa etária. Em estudo que avaliou escolares de 7 a 14, anos observou que meninos aos 14 anos são significativamente mais altos que as meninas (PETROSKI; SILVA; PELEGRINI, 2008). Este crescimento rápido pode relacionar-se com o estirão puberal que é o pico máximo de crescimento, e serve como auxílio na avaliação do estado nutricional. Barbosa, Franceschini e Priore (2006) ressaltam, inclusive, que o estadiamentopuberal pode interferir em modificações antropométricas e de composição corporal. A evolução da puberdade está associada às mudanças na composição corporal, na massa muscular em vários componentes da aptidão física (LINHARES et al., 2009), Canhadas et al. (2010) afirmam que esta velocidade de crescimento está provavelmente associada com o nível de treinamento intensivo, a seleção antes do treino de futebol ou as atividades físicas realizadas por estas crianças e adolescentes.

No presente estudo, observa-se que a maioria dos meninos (97\%) encontra-se com 
o peso ideal. A Pesquisa de Orçamento Familiar (POF) 2008-2009, através de uma observação atenta, identificou que a curva de evolução do peso mediano das crianças brasileiras ultrapassa o padrão esperado em relação ao padrão internacional, independentemente da idade e do sexo (BRASIL, 2010).

No presente estudo, o qual faz a junção das dobras cutâneas subescapular e triciptal, para se obter o percentual de gordura, encontra-se um aumento significativo nas faixas etárias dos 9 aos 10 anos de idade. Já no estudo de Canhadas et al. (2010) tal diferença encontra-se apenas nos meninos jogadores de futebol de 11 anos de idade. As dobras subescapular e triciptal refletem o percentual de gordura corporal. De acordo com a classificação de Slaughteret al. (1988)os meninos estudados encontram-se dentro dos padrões adequados para o percentual gordura. No estudo de Vasquez e colaboradores que avaliou jovens e crianças de 6 a 14 anos praticantes de um programa de atividade física regular concluiu que há um efeito positivo da atividade física regular na redução de gordura corporal das crianças e jovens (FERNANDESet al., 2008).

Na POF 2008-2009 a prevalência de déficit de altura na faixa etária de 5 a 9 anos mostrou forte diminuição com o aumento da renda. E as prevalências de excesso de peso e de obesidade aumentaram com a renda nos dois sexos. Nos adolescentes com idade de 10 a 19 anos analisados a prevalência de excesso de peso e obesidade mostrou forte aumento com a renda (BRASIL, 2010).Fernandes et al. (2008), também encontrou chances aumentadas de jovens pertencentes à classe $\mathrm{A}$ serem obesos. Percebe-se associação significativa entre classe socioeconômica e a prevalência de sobrepeso e obesidade. Em contraste com a POF2008-2009 (BRASIL, 2010) e o estudo de Fernandes et al. (2008), a variável peso foi a única a apresentar diferença significativa em relação à classe econômica na atual pesquisa. Sendo os meninos da classe econômica $\mathrm{C}$ com maior peso em comparação com os meninos das classes A e B.

Num estudo realizado por Guimarães et al. (2012), que avaliou a associação de fatores biopsicológicos, socioeconômicos e comportamentais com o excesso de peso e obesidade em escolares, foi verificado que crianças que frequentavam escolas da rede pública pertencentes à classe social $\mathrm{B}$ apresentaram maior frequência de sobrepeso e obesidade quando comparadas com crianças de classe social superior que estudavam na rede privada.

Dentre os fatores relacionados no estudo, a pouca prática de atividades esportivas, associada ao acesso facilitado aos alimentos com elevada densidade energética parecem predominar neste grupo. Soma-se a isso, o uso de tecnologias que facilitam a ocupação do tempo de lazer com atividades 
sedentárias (SILVA; BALABAN; MOTTA, 2005).

Em estudo com 6.965 jovens de 6 a 11 anos e 6.671 de 12 a 17 anos, demonstraram que a prevalência de obesidade aumenta em 2,0\% para cada hora adicional que os mesmos assistem à TV(DIETZ; GORTMAKER, 1985).

Análises recentes sobre a tendência secular da desnutrição infantil no Brasil indicam que o excepcional declínio do problema na década de 2000 ocorreu associado às melhorias observadas no poder aquisitivo das famílias de menor renda, na escolaridade das mães e na cobertura de serviços básicos de saúde e saneamento (BRASIL, 2010). As causas subjacentes ao notável declínio da desnutrição infantil no Brasil parecem residir em melhorias na cobertura de serviços públicos essenciais e em aumentos da renda familiar, ambos favorecendo especialmente os mais pobres (MONTEIRO, 2009).

O excesso de peso e a obesidade expressam o desequilíbrio entre a ingestão e utilização de calorias no corpo humano, chamando a atenção para o aumento da incidência de várias doenças crônicas (como diabetes, doenças do coração e certos tipos de câncer). Para tanto deve-se procurar mudanças nos padrões de alimentação e atividade física da população. E os governos nacionais necessitam promover políticas públicas e ações intersetoriais que vão além de informar e educar os indivíduos. Tais políticas devem, essencialmente, propiciar um ambiente que estimule, apoie e proteja padrões saudáveis de alimentação e de atividade física (BRASIL, 2010).

Os resultados desta pesquisa fazem menção à pesquisa de Canhadas et al. (2010) que teve objetivo e população semelhantes. Entretanto, a atual pesquisa, além de avaliar as características antropométricas realizou a avaliação econômica e relacionou essas duas variáveis. Assim sendo, a presente pesquisa traz novos resultados, pois compara o poder aquisitivo com o estado nutricional, acrescentando uma proposta diferente para novos estudos.

\section{CONCLUSÃO}

No presente estudo encontra-se o predomínio de eutrofia e classe econômica alta. Os meninos da classe econômica mais baixa encontram-se com peso mais elevado quando comparados aos de classe econômica mais alta. Sugere-se ainda que tal fator seja analisado com cautela, uma vez que os estudos populacionais apontam resultados opostos.

\section{REFERÊNCIAS}

1. ASSOCIAÇÃO BRASILEIRA DE EMPRESAS DE PESQUISA (ABEP). Critério de Classificação Econômica Brasil (2010). Disponível em: <http://www.abep.org/criterio-brasil >. Acesso em: 18 abr. 2012. 
2.AZEVEDO, M. R. et al. Tracking of physical activity from adolescence to adulthood: a population-based study.

RevSaude Publica, São Paulo, v. 41, n. 1, p. 69-75, 2007.

3. BARBOSA, K. B. F.; FRANCESCHINI, S. C. C.; PRIORE, S. E. Influência dos estágios de maturação sexual no estado nutricional, antropometria e composição corporal de adolescentes. Rev. Bras. Saúde Matern. Infant., Recife, v. 6, n.4, p. 375-382, 2006.

4. BRASIL. Ministério da Saúde. Ministério do Planejamento, Orçamento e Gestão. Instituto Brasileiro de Geografia e Estatística - IBGE. Pesquisa de Orçamentos familiares 2008-2009. 2010. Disponível em: <http://www.ibge.gov.br/home/estatistica/pop ulacao/condicaodevida/pof/2008_2009_encaa/ pof_20082009_encaa.pdf >. Acesso em 10 mar. 2012.

5. BRASIL. Vigilância alimentar e nutricional (SISVAN): orientações básicas para coleta, processamento, análise de dados e informação em serviços de saúde. Brasília: Ministério da Saúde, 2004. Disponível em: <http://189.28.128.100/nutricao/docs/geral/ori entacoes_basicas_sisvan.pdf $>$. Acessoem: 28 jun. 2012.

\section{BRITO, S.; DREYER, E. Terapia}

Nutricional:Condutas do Nutricionista. GAN/EMTN - HC, Campinas: UNICAMP, 2003. Disponível em:

<http://www.doencasdofigado.com.br/manual nutricionista_na\%20UNICAMP\%20SP.pdf $>$

. Acesso em 08 mai. 2012.

7. BRODERICK, C. R.; WINTER, G. J.; ALLAN, R. M. Sport for special groups. Med J Aust., Sydney, v. 184, n. 6, p. 297-302, 2006.

8. CANHADAS, I. L. et al. Anthropometric and physical fitness characteristics of Young male soccer players. RevBrasCineantropom Desempenho Hum, Florianópolis, v. 12, n. 4, p. 239-245, 2010.
9. CONDE, W. L.; MONTEIRO, C. A.

Valores críticos do índice de massa corporal para classificação do estado nutricional de crianças e adolescentes brasileiros.J Pediatr, Rio de Janeiro, v. 82, n. 4, p. 266-272, 2006.

10. DELLAGRANA, R. A. et al. Estado nutricional e desempenho motor de crianças praticantes de handebol. Fit Perf J,Rio de Janeiro, v. 9, n. 1, p. 72-77, 2010.

11. DIETZ, W. H.; GORTMAKER, S. L. Do we fatten our children at the television set? Obesity and television viewing in children and adolescents. Pediatrics, Illinois, v. 75, n.5, p. 807-812, 1985.

12. EISENSTEIN, E. et al. Nutrição na Adolescência. J Pediatr, Rio de Janeiro, v. 76, Supl. 3 p. S263-S274,2000.

13. FERNANDES, R. A.et al. Riscos para o excesso de peso entre adolescentes de diferentes classes socioeconômicas.

RevAssocMedBras, São Paulo, v. 54, n. 4, p. 334-338, 2008.

14. GUIMARÃES, A.C.A. et al. Excesso de peso e obesidade em escolares: associação com fatores biopsicológicos, socioeconômicos e comportamentais.

ArqBrasEndocrinolMetab, São Paulo. v. 56, n. 2, p. 142-148, 2012.

15. HEYWARD, V. H.; GIBSON, A. L.Advanced fitness assessment and exercise prescription.Champaign:

HumanKineticsPublishers. 1991. 552 p.

16. JELLIFFE, D. B. Evaluaciondel estado de nutrición de lacomunidad. Organizacion

Mundial de la Salud, Ginebra, 1968.

17. LINHARES R. V. et al. Efeitos da maturação sexual na composição corporal, nos dermatóglifos, no somatótipo e nas qualidades físicas básicas de adolescentesArqBrasEndocrinolMetab, São Paulo, v.53, n.1, p. 47-54, 2009. 
18. LOHMAN, T. G. The use of skinfold to estimate body fatness on children and youth. JPERD, Reston, v. 58, n. 9, p. 98-102, 1987.

19. MONTEIRO, C. A. A queda da desnutrição infantil no Brasil. Cad Saúde Pública, Rio de Janeiro, v. 25, n. 5, p. 950951, 2009.

20. ONIS, M. et al. Development of a WHO growth reference for school-aged children and adolescents.Bulletin of the World Health Organization, Geneva, v. 85, p.660-667, 2007.

21. PETROSKI, E. L.; SILVA, R. J. S.; PELEGRINI, A. Crescimento físico e estado nutricional de crianças e adolescentes da região de Cotinguiba, Sergipe.Rev. Paul. Pediatr, São Paulo, v. 26, n. 3, p. 206-211, 2008.

22. SALES, W. F.; MOREIRA, O. C. Capacidade cardiorrespiratória e composição corporal de estudantes participantes e não participantes de aulas de educação física.Braz J Biomotricity, Nova Iguaçu,v. 6, n. 3, p. 153-158, 2012.

23. SILVA, G. A. P.; BALABAN, G.; MOTTA, M. E. F. A. Prevalência de excesso de peso e obesidade em crianças e adolescentes de diferentes condições socioeconômicas. RevBrasSaudeMater

Infantil, Recife, v. 5, n.1, p. 53-59, 2005.

24. SLAUGHTER, M. H. et al. (1988) Skinfold equationsfor estimation of body fatness in children andyouth. Human Biology,Michigan, v. 60, p. 709-723, 1988.

25. ULBRICH, A.Z. et al. Aptidão física em crianças e adolescentes de diferentes estágios maturacionais. Fit Perf J., Rio de Janeiro, v. 6, n. 5, p. 277-282, 2007.

26. VANNUCCHI, H.; UNAMUNO, M. R. DEL L.; MARCHINI, J. S. Avaliação do Estado Nutricional. Medicina, Ribeirão Preto, v. 29, p. 5-18, 1996.

27. VITOLO, M. R. et al. Avaliação de duas classificações para excesso de peso em adolescentes brasileiros. RevSaude Publica, São Paulo, v. 41, n. 4, p. 653-656, 2007.

28. World Health Organization (WHO). Infants and children. In: Physical status: The use and interpretation of anthropometry. Geneva: WHO, n. 854, 1995. Disponível em: $<$ http://apps.who.int/iris/bitstream/10665/370 03/1/WHO_TRS_854.pdf>. Acesso em: 12 mar. 2012. 